\title{
Adhatoda Vasica attenuates inflammatory and hypoxic responses in preclinical mouse models: potential for repurposing in COVID-19-like conditions
}

\section{Atish Prabhakar Gheware}

CSIR-IGIB: CSIR Institute of Genomics \& Interactive Biology - South Campus

Dhwani Dholakia

CSIR-IGIB: CSIR Institute of Genomics \& Interactive Biology - South Campus

\section{S Kanan}

CSIR-CLRI: Central Leather Research Institute CSIR

\section{Lipsa Panda}

CSIR-IGIB: CSIR Institute of Genomics \& Interactive Biology - South Campus

\section{Ritu Rani}

CSIR-IGIB

Bijay Ranjan Pattnaik

CSIR-IGIB

\section{Vaibhav Jain}

CSIR-IGIB

\section{Yash Parekh}

CCMB: Centre for Cellular and Molecular Biology CSIR

\section{M.Ghalib Enayathullah}

CCMB: Centre for Cellular and Molecular Biology CSIR

Kiran Kumar Bokara

CCMB: Centre for Cellular and Molecular Biology CSIR

V Subramanian

CSIR-CLRI: Central Leather Research Institute CSIR

Mitali Mukerji

CSIR-IGIB

\section{Anurag Agrawal}

CSIR-IGIB: CSIR Institute of Genomics \& Interactive Biology - South Campus Bhavana Prasher ( $\sim$ bhavana.p@igib.res.in )

CSIR-IGIB: CSIR Institute of Genomics \& Interactive Biology - South Campus https://orcid.org/00000003-4185-4400 
Research

Keywords: Hypoxia, fibrosis, sepsis, angiogenesis, blood coagulation, SARS-CoV2, COVID-19, Adhatoda Vasica.

Posted Date: February 9th, 2021

DOI: https://doi.org/10.21203/rs.3.rs-105233/v2

License: (c) (i) This work is licensed under a Creative Commons Attribution 4.0 International License.

Read Full License

Version of Record: A version of this preprint was published at Respiratory Research on April 6th, 2021. See the published version at https://doi.org/10.1186/s12931-021-01698-9. 


\section{Abstract}

Background: COVID-19 pneumonia has been associated with severe acute hypoxia, sepsis-like states, thrombosis and chronic sequelae including persisting hypoxia and fibrosis. The molecular hypoxia response pathway has been associated with such pathologies and our recent observations on antihypoxic and anti-inflammatory effects of whole aqueous extract of Adhatoda Vasica (AV) prompted us to explore its effects on relevant preclinical mouse models.

Methods: In this study, we tested the effect of whole aqueous extract of $A V$, in murine models of bleomycin induced pulmonary fibrosis, Cecum Ligation and Puncture (CLP) induced sepsis, and siRNA induced hypoxia-thrombosis phenotype. The effect on lung of $A V$ treated naïve mice was also studied at transcriptome level. We also determined if the extract may have any direct effect on SARS-CoV2 replication

Results: Oral administration AV extract attenuates increased airway inflammation, levels of transforming growth factor-b1 (TGF-b1), IL-6, HIF-1a and improves the overall survival rates of mice in the models of pulmonary fibrosis and sepsis and rescues the siRNA induced inflammation and associated blood coagulation phenotypes in mice. We observed downregulation of hypoxia, inflammation, TGF-b1, and angiogenesis genes and upregulation of adaptive immunity-related genes in the lung transcriptome . AV treatment also reduced the viral load in Vero cells infected with SARS-CoV2.

Conclusion: Our results provide a scientific rationale for this ayurvedic herbal medicine in ameliorating the hypoxia-hyperinflammation features and highlights the repurposing potential of AV in COVID-19-like conditions.

\section{Background}

Increased alveolar hypoxic response levels are inevitable consequences of many respiratory disorders such as chronic obstructive pulmonary disease and pulmonary fibrosis [1,2]. The key player of cellular response to hypoxia is the hypoxia-inducible factor (HIF)-1a and its regulatory protein, the prolyl hydroxylase domain (PHD)-2 enzyme [3]. The induction of HIF-1a is considered to be pro-inflammatory. It leads to transcriptional activation of essential genes implicated in airway remodelling and inflammation, such as vascular endothelial growth factor, transforming growth factor-beta 1 (TGF-b1), inducible nitric oxide synthase, interleukin -17 (IL-17), and IL-6 [3,4]. Thus, it is not just a consequence of diseases, elevated tissue/cellular hypoxia actively participates in exaggerating the inflammatory response contributing to progressive lung damage/injury.

HIF-1a also plays a pivotal role in infection, especially in promoting viral and bacterial replication [5]. In the present COVID-19 pandemic caused by the severe acute respiratory coronavirus 2 (SARS-CoV2), the role of hypoxia response in inducing severe lung inflammation and other outcomes has been one of the most highlighted observations [6-10]. Clinically, the interaction of the host and SARS-CoV2 is broadly described in three stages: first, asymptomatic state; second, a non-severe symptomatic state 
characterized by upper airway and conducting airway response; third, severe respiratory symptomatic state with the presence of hypoxia, acute respiratory distress syndrome (ARDS) and progression to sepsis [7]. During incubation and non-severe state, a specific humoral and cell-mediated adaptive immune response is required to eradicate the virus and prevent disease progression to a severe condition. Thus, strategies to boost immune responses at this stage are undoubtedly important $[7,11]$. However, defective immune response causes further accumulation of immune cells in the lungs, progressing to aggressive production of a pro-inflammatory cytokine such as IL-6, TNF-a resulting in an influx of immune cells and cytokines that damage the airways/ lung architecture. This extended release of cytokines by the immune system in response to the viral infection and/or secondary infections causes severe inflammation, endothelial dysfunction, sepsis and multi-organ damage [7,11,12]. In addition, recent research also reports coagulation abnormalities in severe COVID-19 cases [13]. The relation of hypoxia-coagulation is well known, where we and others also showed the crucial role of cellular hypoxic response in the form of thrombosis and bleeding susceptibility through HIF-1 $a$ and vWF axis [14,15]. Thus, medicinal agents that possess immune-boosting and anti-hypoxic effects could hold a promise for a better therapeutic option to preclude the SARS-CoV2 infection and severity.

We have recently shown an extract of Adhatoda Vasica (AV); an ayurvedic medicine that possesses robust anti-hypoxic properties and can reduce severe airway inflammation induced by an augmented hypoxic response in treatment-resistant asthmatic mice [16]. The anti-HIF-1a effect of AV also restores the cellular hypoxia-mediated loss of mitochondrial morphofunction in vitro [16]. As a follow-up, we evaluated AV's usefulness in other severe lung pathologies, where hypoxia signalling is pertinent, and which are relevant to the clinical course of COVID-19 namely lung injury, fibrosis, and thrombosis. Since viral proliferation may be altered by molecular modulation of such pathways, we further tested the potential of AV in limiting SARS-CoV2 proliferation .

\section{Methods}

\section{Preparation of plant extract and LC-MS fingerprinting:}

Adhatoda Vasica (AV) was collected from Delhi-NCR region, India in the flowering season (November to March). Water extract of plant (leaves, twigs and flowers) was prepared according to classical method described for rasakriya in Caraka Samhita [17]. The process for the formulation involved preparation of decoction condensation and drying as described in earlier study [18]. Chemical fingerprinting of prepared AV extract was carried out by LC-MS at SAIF, CSIR-CDRI, Lucknow, India.

\section{Animals}

The study was designed and performed following guidelines of the Committee for the Purpose of Control and Supervision of Experiments on Animals (CPCSEA) and approved by Institutional Animal Ethics Committee of CSIR-Institute of Genomics \& Integrative Biology (IGIB), New Delhi, India. The BALB/c and C57BL/ 6 male mice (8-10 weeks old) were bred under the pathogen-free condition. They were acclimatized to animal house environment one week before starting the experiments at CSIR-IGIB, New 
Delhi, India and maintained according to guidelines of CPCSEA. All the surgical procedures were performed under sodium pentobarbital anaesthesia and maximum efforts are taken for minimum suffering of animals.

\section{Grouping and treatment of mice}

Mice were mainly divided into two groups as Vehicle and treatment according to the experiment. In case of Cecum ligation puncture (CLP) experiment $(n=5-9), B A L B / c$ mice were divided in Vehicle ( distil water and $10 \%$ ethanol, oral) and CLP (mice underwent CLP surgery). CLP mice subdivide in CLP+Cyclo $A$ (Cyclosporin A treated CLP mice) and CLP+AV-D2 (Adhatoda extract treated CLP mice) group. For CLP experiment, treatment of AV $(130 \mathrm{mg} / \mathrm{kg}$ dissolved in distilled water, oral) or Cyclo A ( Cyclosporin A, $15 \mathrm{mg} / \mathrm{kg}$ dissolved in $10 \%$ ethanol, orall) was started two days (48hours) before CLP and was continued till the mice survives after CLP (figure 1$)$. In that some mice $(n=3-4)$ from each group were sacrificed after 20 hours of CLP to assess lung histology and cytokine levels. Similarly, in bleomycin fibrosis model ( $n=$ 5), C57BL/6 mice were divided in Vehicle (i.e. Sham), Bleo (bleomycin treated) and Bleo+AV-D2 (AV $130 \mathrm{mg} / \mathrm{kg}$ treated Bleo mice). In that AV treatment was done from day 18 to 21 , as shown in the schematic (figure $1 \mathrm{~A}$ ). Bleomycin (3.5 U/ kg of mice) was given intratracheally to isoflurane-anesthetised $\mathrm{C} 57 \mathrm{BL} / 6$ mice on day 0 of the protocol (figure 1) to induce fibrotic changes in mice as described previously [19]. For transcriptomic research $(n=4-5), B A L B / c$ mice divided into Vehicle (distil water, oral) and Adhatoda Vasica (AV) extract group. AV group is further subdivided into two according to its dose: AV-D2 (Adhatoda Vasica extract $130 \mathrm{mg} / \mathrm{kg}$, dissolved in distilled water, oral) and AV-D4 (Adhatoda Vasica extract $260 \mathrm{mg} / \mathrm{kg}$, dissolved in distilled water, oral) as described previously [16]. Distil water or AV $(130 \mathrm{mg} / \mathrm{kg}$ or $260 \mathrm{mg} / \mathrm{kg}$ ) treatment was given to mice by oral gavage for consecutive four days as represented in the figure. In PHD2 siRNA induced hypoxia model $(n=4-5)$ BALB/c mice were divided in scrambled siRNA (Scrm siRNA), prolyl hydroxylase domain-2 siRNA (PHD2 siRNA) and AV-D4 treated PHD2 siRNA group (PHD2 siRNA+AV-D4) group. AV-D4 dose $(260 \mathrm{mg} / \mathrm{kg}$, dissolved in distilled water, oral)

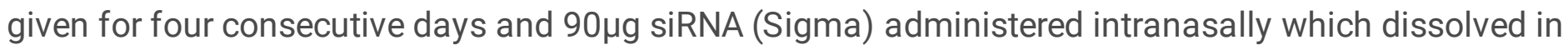
ultrapure DNase and RNAse free water with in-vivo jetPEI as the transfection reagent (Polyplus Transfection, France) to isoflurane-anesthetised mice on day 1,3 and 5th of the protocol.

\section{Clotting and bleeding time assay, blood collection and platelet measurement}

Tail bleeding was measured as described previously [15]. Briefly, anesthetized mice's tail amputated with a sharp scalpel, and bleeding time was then determined by monitoring the duration of animal tail bleeding until it ceased and was kept in a prone position and immersed in PBS. Clotting time measured by the capillary tube method. The mice's tail was cleaned with $70 \%$ alcohol and punctured with a $1 \mathrm{ml}$ syringe needle. Filled two capillary tubes with free-flowing blood from the puncture site after wiping the first drop of blood. Stop clock started and capillary tubes were broken to see whether a thin fibrin stand formed between two broken ends. After fibrin stand is observed, clotting time measured from the average of two capillary tubes. For platelet measurement, blood obtained by cardiac puncture and collected in EDTA coated MiniCollect tubes (Greiner Bio-One Gmbh, kremsmünster, Austria) as described [15]. The 
whole blood was used to measure total and of active Platelet count and was carried out through flow cytometry using FACSCalibur (BD Biosciences, USA). Briefly, diluted whole blood (1:4) in PBS was incubated with APC conjugated anti-CD62P (eBioscience Inc, San Diego, CA, USA) and FITC conjugated anti-CD41 (eBioscience Inc, San Diego, CA, USA) for 15 min. Matched fluorescein-conjugated isotype control antibodies were used simultaneously for staining for comparison. The activity was compared using CellQuest Pro software (BD Biosciences, USA).

\section{Bronchoalveolar lavage fluid collection and histopathology}

Bronchoalveolar lavage fluid (BAL) was collected by instilling $1 \mathrm{ml}$ PBS into the tracheotomised airway and recovered BAL fluids were processed to get total leukocyte count, as described previously (). For lung histology, the lungs were excised and fixed in $10 \%$ buffered formalin. The fixed, paraffin-embedded tissues cut into 5um sections and either stained with hematoxylin and eosin (H\&E) to asses inflammation or Masson's trichome (MT) staining to assess collagen content.

\section{Cecum ligation puncture (CLP) procedure}

Mice were anesthetised by injecting intraperitoneally a solution of $1: 1$ ketamine $(75 \mathrm{mg} / \mathrm{kg})$ and xylazine $(15 \mathrm{mg} / \mathrm{kg})$. The abdomen was shaved, and the peritoneum area was disinfected betadine solution followed by wiping with a $70 \%$ alcohol. Under aseptic conditions, a $1 \mathrm{~cm}$ midline incision was made, and the cecum carefully exposed with the adjoining intestine. The cecum was then tightly ligated with a 3.0 Mersilk (PROLENE, 8680G; Ethicon) sutures at the base and punctured once with a 19-gauge needle on the same side of the cecum. A small amount of stool extruded to ensure patency of the puncture sites. The cecum then returned to the peritoneal cavity, and the wound was closed with 3.0 Mersilk sutures. Control mice (i.e. Sham), the cecum was exposed out and then returned to the peritoneum without ligation or puncture. Mice were resuscitated by injecting subcutaneously $1 \mathrm{ml}$ of pre-warmed $0.9 \%$ saline solution using a $25 \mathrm{G}$ needle. After surgery, animals placed immediately to a cage with exposure to a heating lamp of $150 \mathrm{~W}$ until they recovered from the anaesthesia. The recovery time is from $30 \mathrm{~min}$ to 1 hour. Mice were monitored every 12 hours for survival or euthanised after 20 hours $(n=3)$ for measurement of cytokines while they were fed with their regular diet and water. Two independent experiments recorded the mortality of mice after CLP surgery.

\section{TGF-b, IL-6,IFN-g, HIF-1a, PHD2, and vWF measurement}

The levels of TGF-b, IL-6, IFN-g (BD, USA), HIF-1a (R\&D, USA), PHD2 ((USCN, China) and vWF (USCN, China) were measured in lung tissue homogenate or in plasma of the mice by sandwich ELISA, as per manufacturer's protocol.

\section{RNA isolation and whole transcriptome analysis:}

Total RNA was isolated from mouse lung tissue treated with AV (AVD2 and AV-D4) or distilled water (vehicle) using the RNeasy Plus Mini Kit (Qiagen, CA, USA) following the manufacturer's protocol. For genome-wide expression analysis, the Affymetrix GeneChip MTA 1.0 array was used according to the 
manufacturer's instruction. For each sample, $250 \mathrm{ng}$ of RNA was quantified and hybridized to microarray chips following a series of consecutive steps described in the protocol. After hybridization, microarray chips are then scanned using an Affymetrix GCS 3,000 scanner (Affymetrix, CA, USA) and the signal values are further evaluated using the Affymetrix ${ }^{\circledR}$ GeneChip ${ }^{\text {TM }}$ Command Console software. Raw data automatically extracted using the Affymetrix data extraction protocol in the Affymetrix GeneChip ${ }^{\circledR}$ Command Console ${ }^{\circledR}$ Software (AGCC). CEL file import, mRNA level, all analysis, and export of the results were all performed using Affymetrix ${ }^{\circledR}$ Expression Console ${ }^{\mathrm{TM}}$ software. A comparative study between the vehicle and the $A V$ treated samples done by using fold-change and $p$-value, genes considered to the differentially expressed by applying the criteria of significance p-value less than or equal to 0.05 .

\section{Functional enrichment and Connectivity map analysis}

For functional analysis, we used Enrichr (amp.pharm.mssm.edu) tool. For pathway and gene ontology analysis, we examined gene enrichment in Cellular Compartment, Biological Processes, BioPlanet, Wiki, KEGG human pathway and gene set enrichment was considered if P-value less than 0.05 in Enrich $r$ tool. For connectivity map (CMap) analysis, differentially expressed genes ranked according to fold change and list of top 150 up and down-regulated genes compatible with the CMAP data signatures was used to query the connectivity using clue.io touchstone database. A positive score in CMap analysis indicates a similar expression pattern of AV with compared compounds' gene expression signature, whereas a negative score indicates an opposite pattern.

\section{Molecular docking}

The complete genome sequence of the novel SARS-CoV-2 virus was obtained from the National Centre for Biotechnology Information (NCBI) nucleotide database (NC_045512.2). The available 3D crystal structures of all the target proteins such as 3CLpro, PLpro, RdRp, S-protein, ACE2 and JAK2 were taken from protein data bank [20]. Others structures (NSP4, NSP7, NSP8, NSP9, NSP13, NSP14, NSP15 and NSP16, and TMPRSS2) were built using homology modelling with suitable templates using Swiss model [21] and I-TASEER web-servers [22]. The active regions of the proteins were identified by COACH metaserver and the results were compared with results from CASTp web server [23]. The anti-COVID-19 activity of the compounds extracted from the Adhatoda vasica were investigated using Molecular Docking studies using Schrodinger suite (Maestro) [24] and AutoDock vina packages [25]. In Schrodinger suite, all the target proteins were prepared using protein preparation wizard that included optimization followed by minimization of heavy atoms of proteins. The energy minimized 3D structures of all the ligands were prepared using LigPrep. The best pose of ligands that fit well in the protein cavity was carried out using OPLS3 force field with Glide package in Extra Precision mode (XP) mode. According to the size of binding cavity of the proteins, the coordinates $\mathrm{x}, \mathrm{y}$ and $\mathrm{z}$ of the grid box were chosen with the grid resolution of 1 $\AA$ for calculations using AutoDock vina package.

\section{Cellular model, drug treatment and detection of SARS-CoV-2 using a qPCR assay}


Vero cells were maintained in Dulbeco Minimum Essential Medium (Gibco) containing 10\% Fetal Bovine Serum (Gibco) at $37^{\circ} \mathrm{C}, 5 \% \mathrm{CO}_{2}$. Cells were seeded into 96 -well tissue culture plates $24 \mathrm{~h}$ prior to infection with SARS-CoV2 (Indian/a3i clade/2020 isolate) in BSL3 lab of CSIR-CCMB. The effect of (AV) aqueous extract was tested against the SARS-CoV2 virus [26] by taking different concentrations of AV: 100, 50, $12.5,6.25(\mu \mathrm{g} / \mathrm{mL})$ in DMEM media. Briefly, the cells were primed with the AV for 2 hours. The virus inoculum $(0.1 \mathrm{MOI})$ was added to the cells along with different concentrations of $A V$ and were left for infection for 3 hours. Post-infection, viral inoculum was replaced with fresh media containing 10\% FBS and were maintained at $37^{\circ} \mathrm{C}, 5 \% \mathrm{CO}_{2}$ until 72 hours. After 72 hours, cell supernatant was collected and spun for $10 \mathrm{~min}$ at $6,000 \mathrm{~g}$ to remove debris and the supernatant was transferred to fresh collection tubes. RNA was extracted from $200 \mu \mathrm{L}$ aliquots of sample supernatant using the MagMAX ${ }^{\mathrm{TM}}$ Viral/Pathogen Extraction Kit (Applied Biosystems, Thermofisher). Extraction of viral RNA was carried out according to the manufacturer's instructions. The viral supernatants from the test groups were added into the deep well plate (KingFisher ${ }^{\mathrm{TM}}$ Thermo Scientific) along with a lysis buffer containing the following components - MagMAX ${ }^{\mathrm{TM}}$ Viral/Pathogen Binding Solution; MVP-II Binding Beads; MagMAX ${ }^{\mathrm{TM}}$ Viral /Pathogen Proteinase-K of $260 \mu \mathrm{L} ; 10 \mu \mathrm{L} ; 5 \mu \mathrm{L}$ respectively for $200 \mu \mathrm{L}$ of sample. RNA extraction was performed using KingFisher Flex (version 1.01, Thermo Scientific) by following manufactures instructions. The eluted RNA was immediately stored in -80冈C until further use. The detection of SARSCoV2 was done using COVID-19 RT-PCR Detection Kit (Fosun 2019-nCoV qPCR, Shanghai Fosun Long March Medical Science Co. Ltd.) according to the manufacturer's instructions. The kit detects Envelope gene (E; ROX labelled), Nucleocapsid gene ( $\mathrm{N}$ - JOE labelled) and open reading frame1ab (ORF1ab, FAM labelled) specific to SARS-CoV2 for detection and amplification of the cDNA. SARS-CoV-2 cDNA (Ct 28) was used as a positive control. The log viral particles and a semi-log graph was plotted through the linear regression equation obtained using the RNA extracted from the known viral particles by RT-qPCR, using $\mathrm{N}$ - and ORF1 ab genes specific to SARS CoV2 virus and percent viral reduction was calculated, as described previously [27]. However, $\mathrm{Ct}$ value of $\mathrm{N}$ gene is considered to calculate the \% viral reduction [27].

\section{Statistical analysis}

Statistical significance determined by one-way analysis of variance and analysis was done using GraphPad Prism software. In the case of mice experiment, all data represent mean $\pm S E M ; n=3-10$ in each group and significance denoted by ${ }^{*}<<0.05$, ${ }^{\star *} p<0.01$, ${ }^{\star \star *} p<0.001$. $p$-value $>0.05$ is considered non-significant (NS). Significance of the survival study determined by Log-rank (Mantel-Cox) test using GraphPad Prism software.

\section{Results}

\section{AV treatments inhibits the bleomycin induced pulmonary fibrosis features as well as increased HIF-1a levels in mice}

To test the effect of $A V$ treatment on lung fibrosis, bleomycin treated mice were orally administered with AV $(130 \mathrm{mg} / \mathrm{kg}, A V-D 2)$ as shown in figure $1 \mathrm{~A}$. We observed a significant increase in TGF- $\beta 1$ and HIF-1a 
levels in bleomycin (Bleo) treated mice lung than control-Sham mice, which decreased after AV-D2 treatment (figure 1B, C). Masson's trichrome staining showed a marked increase in collagen deposition in Bleo mice lungs compared to Sham mice (figure 1D). AV-D2 treatment reduces this increased collagen deposition in Bleo mice (figure 1D).

\section{AV ameliorates the hallmarks of lung inflammation and injury in mice model of sepsis}

Mice that underwent CLP (Cecal ligation and puncture) surgery did not show significant increase in HIF1a levels after 20 hours of surgery (figure 1F). Still, its downstream target, such IL-6, was significantly increased (figure 1G), and IFN-g was decreased (figure 1H) in lung homogenate after 20 hours of surgery compared to sham mice. AV pre-treatment restored the levels of both cytokines in mice lungs, whereas Cyclo-A (a positive control) pre-treatment reduced only IL-6 levels in mice (figure 1G, H). Besides, histological analyses showed that CLP mice lung sections stained with haematoxylin and eosin (H\&E) had increased inflammation and blood exudation (figure 1I). Pre-treatment of AV-D2 and Cyclo A to CLP mice showed reduced inflammation and blood exudation in lung histological sections (figure 1I). CLP surgery also leads to a significant decrease in mice survival rate compared to sham group (figure $1 \mathrm{~J}$ ). Treatment of Cyclo-A or AV-D2 to CLP mice significantly increases their survival rate compared to CLP untreated mice (figure $1 \mathrm{~J}$ ). In CLP+Cyclo $A$ and CLP+AV-D2 group, the mice survival rate after 24 hours is 66.6 and $44.4 \%$, respectively (figure $1 \mathrm{~K}$ ). Though, in both groups, survival rate is $33.33 \%$ at the end of 142 hours of CLP surgery (figure $1 \mathrm{~K}$ ).

\section{AV treatment inhibits hemostatic outcomes of hypoxia response induced by PHD2 siRNA in mice}

Next, to test whether anti- HIF-1a effects of AV also prevents the blood coagulation phenotype [15], we treated naïve BALB/c mice with AV-D2 (130 mg/kg) and AV-D4 (260 mg/kg) concentration (figure 2A). Oral administration of AV-D2 and AV-D4 to naïve mice does not cause any significant change in body weight and lung and liver histological architecture (figure 2B, C), indicating its non-toxic nature. In the hemostasis parameter, treatment of AV-D4 dose to naïve healthy BALB/c mice causes a decrease in total as well as activated platelet count. Still, it does not affect mice tail bleeding time (figure 2D, E). To confirm the above-observed effect of AV-D4 on blood parameters, we induce cellular hypoxia response in mice by specific PHD2 siRNA treatment (figure 2F), as described previously [15]. PHD2 siRNA treatment (figure S1A) leads to a significant decrease in blood clotting and tail bleeding time (figure 2G). It also causes an overall increase in total and activated platelet count in mice blood (figure $2 \mathrm{H}$ ). These changes induced by PHD2 siRNA are associated with increased blood HIF-1a and vWF levels, indicating the development of platelet aggregation (figure 2I, J). Interestingly, AV-D4 treatment to PHD2 siRNA mice causes a significant reversal of blood coagulation phenotype in terms of mice's blood clotting time, platelet count (total and active), and vWF levels (figure $2 \mathrm{G}-\mathrm{I}$ ). These effects of $\mathrm{AV}$ are associated with the reversal of increased blood HIF-1 a levels (figure 2J). However, AV treatment does not affect the mice's bleeding time, which was reduced after PHD2 siRNA treatment (figure $2 \mathrm{G}$ ).

\section{Modulation of immune response and hypoxia pathway genes: Revealed from lung transcriptome of AV treated mice}


Lung transcriptomic analysis showed an upregulation of 1258 genes after AV-D4 treatment and 375 gens after AV-D2 treatment in naive mice. While, 1133 genes in AV-D4 and 262 genes in AV-D2 were downregulated, compared to Vehicle (distilled water) treated mice. We observed enrichment of pathways like IL-2 signaling, T cell signaling, T cell-mediated immunity, natural killer cell-mediated cytotoxicity, Haematopoietic cell lineages in the AV-D4 up-regulated genes. Similarly, biological processes like neutrophil activation and degranulation, neutrophil-mediated immunity, immune response regulation, and cellular defense response was enriched in AV-D4 up-regulated genes (figure 3A). In AV-D2 up-regulated genes, pathways relevant in mitochondria, $T$ cell signaling, cytotoxic $T$ cell-mediated immune response are enriched (figure $3 \mathrm{~A}$ ). At the same time, pathways like collagen biosynthesis, extracellular matrix organization, TGF beta regulation, hypoxia, and associated inflammatory MAPK-signaling are significantly enriched in both AV-D4, and AV-D2 downregulated genes (figure 3A). Overall, it indicates that AV treatments favour the expression of genes important in immunity and adaptive immune response and inhibitory to genes involved in hypoxia associated angiogenesis, fibrosis, and inflammatory cascade. These findings of AV might be relevant in COVID-19 treatment, where inhibition of adaptive immune response and induction of hypoxia-inflammatory response seen in SARS-CoV2 infected patients samples [9,10,28-30]. Besides, increased levels of immune cells in bronchoalveolar lavage fluid (BAL) of mice treated with AV, compared to vehicle-treated mice (figure S1B) further support our observation. Furthermore, to identify similarities and differences in AV gene expression pattern with other FDA-approved drugs and bio-actives, we mapped the transcriptomic signature of AV using Connectivity Map (CMap) database. We observed drugs like glucocorticoids, HDAC inhibitors, and NSAIDS agents (that target cyclooxygenase) have a similar gene expression patterns with AV (figure S1C). These drugs or compounds are shown to have therapeutic potential against COVID-19 [31-34]. The possibility of such directs effects was further examined.

\section{In silico and in vitro analysis demonstrates the therapeutic potential of AV against SARS-CoV2}

Chemical components present in Adhatoda Vasica (table S1, S2) were examined by molecular docking analysis with SARS-CoV2 and host proteins. Tables 1 and S1 represent the docking results of the constituents of Adhatoda vasica with key target proteins of the SARS-CoV-2 virus. The compounds such as Luteolin-6,8-di-C-glucoside (-9.91 kcal/mol for 3CLpro, -9.43 for S-protein, -15.25 for NSP14, -11.12 for TMPRSS2, -9.82 for ACE2), Luteolin-6-C-glucoside-8-C-arabinoside (-11.59 kcal/mol for 3CLpro, -14.99 for NSP14, -13.46 for JAK2) and Apigenin-6,8-di-C-arabinoside (-11 kcal/mol for 3CLpro , -12.17 kcal/mol for NSP14, -10.77 for JAK2), Kaempferol_3_O_rutinoside (-8.9 kcal/mol for 3CLpro , -12 kcal/mol for NSP14, -11.77 for JAK2) shows higher docking score values to the viral and host target proteins essential for SARS-CoV2 transcription, replication and infection (table 1 and S1). The key residues of target proteins that contribute more for binding with the compounds of Adhatoda vasica are shown in Table S2. The compound Luteolin-6-C-glucoside-8-C-arabinoside makes cation- $\pi$ interaction with residue (His 41) of $3 \mathrm{CLpro}$ with higher affinity $-11.59 \mathrm{kcal} / \mathrm{mol}$ (figure 3B). The $\pi-\pi$ stacking interaction between the Luteolin6,8-di-C-glucoside and residues (Phe 426 and Phe 506) of NSP14 protein enhances the binding affinity to $-15.25 \mathrm{kcal} / \mathrm{mol}$ when compared to other compounds as shown in figure $3 \mathrm{C}$, and other interaction plots are represented in Supporting material figure S2 and 3. In addition, AV's flavonoids are also shown to 
have a higher binding affinity for proteins involved in hypoxia and inflammation [16]. To confirm the insilico observation, we tested the anti-SARS-CoV2 potential of AV using in vitro model of SARS-CoV2 infection. We observe a $63 \%$ viral reduction after AV treatment $(100 \mu \mathrm{g} / \mathrm{ml})$ in Vero cells infected with SARS-CoV2, compared to the untreated group (figure 4). These results further substantiate the potential of $A V$ in treating COVID-19.

\section{Discussion}

Adhatoda Vasica or Vasa has been extensively used in Ayurveda for treating a wide range of inflammatory and respiratory conditions [35]. Even in modern clinical practice, it is recommended for strong bronchodilatory and antitussive effects [35,36]. AV's active ingredients and their derivates such as Bromhexine, and Ambroxol are effective against various respiratory ailments like asthma, COPD, and tuberculosis [35]. We have recently shown that $A V$ alleviates the severe airway inflammation in steroidnonresponsive asthmatic by inhibition of HIF-1a (key transcription factor in hypoxia) via its negative regulator, $\mathrm{PHD} 2$., AV thereby modulates hypoxic response, which forms the basis for its diverse therapeutic effects including amelioration of mitochondrial dysfunction [16]. In this study we examined anti-hypoxic effects of $\mathrm{AV}$ in other hypoxia-inflammation prevalent conditions such as lung injury, fibrosis, and thrombosis. These are relevant to the current global pandemic of COVID-19, specifically progression and sequelae of SARS CoV2 infection (figure 5).

Our study shows that AV could reverse the pulmonary fibrosis (PF) pathological features in the bleomycin mice model (figure 1). HIF-1a stabilization is observed in many cell types of PF lungs and causes increase in collagen synthesis, fibrosis, TGF- $\beta 1$, VEGF levels, and proliferation of fibroblasts [1,37]. $\mathrm{AV}$ treatment reduces the increased expression of HIF-1 a protein in bleomycin treated mice lungs and attenuates increased TGF- $\beta 1$ and collagen content in mice lungs (figure 1A-D). These preliminary results substantiate the hypoxia modulating effect of $A V$ in chronic lung disease conditions such as fibrosis. There were strong effects of AV treatment on inflammatory lung injury and CLP induced lung injury and mortality in mice was reduced (figure 1I-K). Interestingly, we observed a decrease in IFN-g levels upon CLP in mice, which was restored after AV treatment (figure $1 \mathrm{H}$ ). Our observation with the IFN-g level in CLP mice is not in line with previous studies [38], and it is likely due to the differences in the time of measurement after CLP. We speculate that the mechanism of AV action may not be directly antiinflammatory but rather restoration of appropriate inflammatory responses and blocking of inappropriate inflammation. This would be similar to the clinical findings that support the adjuvant IFN-g immunotherapy concept to improve the host immune response against infection [39,40]. AV treatment also reduced the HIF-1a induced pro-thrombotic state that may additionally be relevant to lung injury and inflammation (figure $2 \mathrm{~F}-\mathrm{J}$ ).

In the course of our study, we realized that the effects of AV on phenotypic features of the lung and systemic inflammation could also prove beneficial for the present pandemic situations. In SARS-CoV2 infection, elevated hypoxia response seems to be a consequence of hyper-inflammation that contributes to disease severity $[6,7]$. We relate the therapeutic relevance of $A V$ for the above-observed effects against 
severe patho-phenotypes associated with the critical stage of COVID-19, characterized by severe lung inflammation, hypoxemia, angiogenesis, sepsis, and altered coagulation profile $[6,8,11-13]$. Therefore, the anti-hypoxic property of $\mathrm{AV}$ would be advantageous in attenuating the critical inflammatory stage of COVID-19. Our transcriptome results of AV treated mice also show down-regulation of genes related hypoxia-inflammation pathway (figure $3 \mathrm{~A}$ ). In view of the multitude of effects of AV that may alter cellular response to infection (Figure 5), we further determined whether there may be a direct or indirect effect of AV on SARS-CoV2 proliferation. We screened AV's chemical components against SARS-CoV2 and host target proteins and found possible interactions with both (table 1, S1, and S2) [41]. The in vitro viral inhibition supports that these may be relevant (figure 4). More studies are warranted to confidently determine whether there is meaningful anti-viral activity against SARS-CoV2.

\section{Conclusion}

Treatment of Adhatoda Vasica extract alters the cellular hypoxic response and modulates the inflammation-thrombosis axis to reduce lung injury, thrombosis and fibrosis. Moreover, in-silico and in vitro analysis suggest its role in preventing viral entry and replication. AV is a suitable candidate for clinical trials in COVID-19 patients.

\section{Abbreviations}

HIF-1a: hypoxia inducible factor-1 a; PHD2: prolyl hydroxylase domain 2; COVID-19: Coronavirus disease2019; AV: Adhatoda Vasica; TGF-b1: transforming growth factor-b1; IL-6: Interleukin-6; IL-17: Interleukin17; IFN-g: interferon gamma; CLP: Cecum Ligation and Puncture; PHD2: Prolyl hydroxylase domain 2; SARS-CoV2: Severe acute respiratory coronavirus 2; PBMC: Peripheral blood mononuclear cell; BALF: Bronchoalveolar lavage fluid; ARDS: Acute respiratory distress syndrome; vWF: von Willebrand factor; Bleo: Bleomycin treated mice; H\&E: hematoxylin and eosin; MT: Masson's trichome; CMap: connectivity map; nsp: non-structural protein; 3CLpro: 3C-like protease; PLpro: Papain-like protease; RdRP: RNAdependent RNA polymerase; S-pro: Spike protein; ACE2: Angiotensin-converting enzyme 2; TMPRSS2: Transmembrane Serine Protease 2; JAK: Janus kinase.

\section{Declarations}

\section{Ethics approval statement}

The mice experiment was designed and performed following guidelines of the Committee for the Purpose of Control and Supervision of Experiments on Animals (CPCSEA) and approved by Institutional Animal Ethics Committee of CSIR-Institute of Genomics \& Integrative Biology (IGIB), New Delhi, India.

In vitro viral culture ethical approval- to add

\section{Consent for publication}


Not applicable

\section{Availability of data and materials}

The datasets used and/or analysed during the current study are available from the corresponding author on reasonable request. The transcriptome data from this study have been submitted to the Gene Expression Omnibus (GEO) under the accession number: GSE156759

Funding: This work is supported by grant to CSIR-TRISUTRA (MLP-901) from the CSIR and Center of Excellence grant by Ministry of AYUSH, Govt. of India.

\section{Competing interests}

The authors declare that they have no competing interests.

\section{Authors'Contributions}

A.G. designed and performed the experiment, analysed the results and wrote the paper. D. D. performed CMap analysis and interpreted the results. S. K. performed molecular docking analysis. L.P., B.R.P. and V.J. contributed to animal model experiments. Y.P., M.G.E. performed in vitro screening against SARSCOV2 culture, R.R. contributed to analysis and manuscript writing, K.K.B. designed, performed, interpreted the in vitro SARS-COV2 culture results and wrote the paper. V.S. designed, performed, interpreted the molecular docking analysis and wrote the paper. B.P. conceptualized the study, provided AV, quality control information, designed the experiment, analysed and discussed the results and wrote the paper. A.A. and M.M. designed experiments, analysed and discussed the results and wrote the paper. All the authors reviewed and approved the final version of the manuscript.

\section{Acknowledgments}

We acknowledge animal house and imaging department for access to facility. We thank Dr. Ramniwas Prasher for his valuable suggestions regarding AV extract preparation and its use in clinical terms of Ayurveda. We also thank Arjun Ray for discussion and suggestion in molecular docking analysis. AG, LP and KK acknowledge AcSIR (Academy of Scientific and Innovative Research) for PhD. registrations and CSIR (Council of Scientific and Industrial Research) for fellowship.

\section{References}

1 Tuder RM, Yun JH, Bhunia A, et al. Hypoxia and chronic lung disease. J. Mol. Med. 2007;85:131724. doi:10.1007/s00109-007-0280-4

2 Fröhlich S, Boylan J, Mcloughlin P. Hypoxia-induced inflammation in the lung: A potential therapeutic target in acute lung injury? Am. J. Respir. Cell Mol. Biol. 2013;48:271-9.

doi:10.1165/rcmb.2012-0137TR 
3 Palazon A, Goldrath AW, Nizet V, et al. HIF transcription factors, inflammation, and immunity. Immunity 2014;41:518-28. doi:10.1016/j.immuni.2014.09.008

4 Watts ER, Walmsley SR. Inflammation and Hypoxia: HIF and PHD Isoform Selectivity. Trends Mol. Med. 2019;25:33-46. doi:10.1016/j.molmed.2018.10.006

$5 \quad$ Eltzschig HK, Carmeliet P. Hypoxia and inflammation. N. Engl. J. Med. 2011;364:656-65. doi:10.1056/NEJMra0910283

6 Lang M, Som A, Mendoza DP, et al. Hypoxaemia related to COVID-19: vascular and perfusion abnormalities on dual-energy CT. Lancet Infect. Dis. 2020. doi:10.1016/S1473-3099(20)30367-4

7 Shi Y, Wang Y, Shao C, et al. COVID-19 infection: the perspectives on immune responses. Cell Death Differ. 2020;27:1451-4. doi:10.1038/s41418-020-0530-3

8 Mason RJ. Pathogenesis of COVID-19 from a cell biology perspective. Eur. Respir. J. 2020;55. doi:10.1183/13993003.00607-2020

9 Codo AC, Davanzo GG, Monteiro L de B, et al. Elevated Glucose Levels Favor SARS-CoV-2 Infection and Monocyte Response through a HIF-1 a/Glycolysis-Dependent Axis. Cell Metab 2020;32:437-446.e5. doi:10.1016/j.cmet.2020.07.007

10 Gibellini L, De Biasi S, Paolini A, et al. Altered bioenergetics and mitochondrial dysfunction of monocytes in patients with COVID-19 pneumonia. EMBO Mol Med 2020;12. doi:10.15252/emmm.202013001

11 Tay MZ, Poh CM, Rénia L, et al. The trinity of COVID-19: immunity, inflammation and intervention. Nat. Rev. Immunol. 2020;20:363-74. doi:10.1038/s41577-020-0311-8

12 Ackermann M, Verleden SE, Kuehnel M, et al. Pulmonary vascular endothelialitis, thrombosis, and angiogenesis in Covid-19. N Engl J Med 2020;383:120-8. doi:10.1056/NEJMoa2015432

13 Jose RJ, Manuel A. COVID-19 cytokine storm: the interplay between inflammation and coagulation. Lancet Respir. Med. 2020;8. doi:10.1016/S2213-2600(20)30216-2

14 Mojiri A, Nakhaii-Nejad M, Phan WL, et al. Hypoxia results in upregulation and de novo activation of von willebrand factor expression in lung endothelial cells. Arterioscler Thromb Vasc Biol 2013;33:1329-38. doi:10.1161/ATVBAHA.113.301359

15 Aggarwal S, Gheware A, Agrawal A, et al. Combined genetic effects of EGLN1 and VWF modulate thrombotic outcome in hypoxia revealed by Ayurgenomics approach. J Trans/ Med 2015;13:184. doi:10.1186/s12967-015-0542-9 
16 Gheware A, Panda L, Khanna K, et al. Adhatoda Vasica ameliorates cellular hypoxia dependent mitochondrial dysfunction in acute and severe asthmatic mice. bioRxiv 2020;:2020.04.01.019430. doi:10.1101/2020.04.01.019430

17 P. S. Charaka Samhita: text with english translation. Varanasi, India: : Chaukambha Orientalia Publisher 1981.

18 Prasher R, Pandey D, De S, et al. Standardization of Vasa Ghrta and its extract form and their comparative Pharmaco-Clinical study with special reference to Swasa Roga. Ayu 1999;6.

19 Das S, Kumar M, Negi V, et al. MicroRNA-326 regulates profibrotic functions of transforming growth factor- $\beta$ in pulmonary fibrosis. Am J Respir Cell Mol Biol 2014;50:882-92. doi:10.1165/rcmb.201301950C

20 Berman HM, Westbrook J, Feng Z, et al. The Protein Data Bank. Nucleic Acids Res. 2000;28:23542. doi:10.1093/nar/28.1.235

21 Waterhouse A, Bertoni M, Bienert S, et al. SWISS-MODEL: Homology modelling of protein structures and complexes. Nucleic Acids Res 2018;46:W296-303. doi:10.1093/nar/gky427

22 Yang J, Yan R, Roy A, et al. The I-TASSER suite: Protein structure and function prediction. Nat. Methods. 2014;12:7-8. doi:10.1038/nmeth.3213

23 Tian W, Chen C, Lei X, et al. CASTp 3.0: Computed atlas of surface topography of proteins. Nucleic Acids Res 2018;46:W363-7. doi:10.1093/nar/gky473

24 Friesner RA, Murphy RB, Repasky MP, et al. Extra precision glide: Docking and scoring incorporating a model of hydrophobic enclosure for protein-ligand complexes. J Med Chem 2006;49:6177-96. doi:10.1021/jm051256o

25 Trott O, Olson AJ. AutoDock Vina: Improving the speed and accuracy of docking with a new scoring function, efficient optimization, and multithreading. J Comput Chem 2009;31:NA-NA. doi:10.1002/jcc. 21334

26 ASTM E1052 - 20 Standard Practice to Assess the Activity of Microbicides against Viruses in Suspension. https://www.astm.org/Standards/E1052.htm (accessed 18 Jan 2021).

27 Caly L, Druce JD, Catton MG, et al. The FDA-approved drug ivermectin inhibits the replication of SARS-CoV-2 in vitro. Antiviral Res 2020;178:104787. doi:10.1016/j.antiviral.2020.104787

28 Xiong Y, Liu Y, Cao L, et al. Transcriptomic characteristics of bronchoalveolar lavage fluid and peripheral blood mononuclear cells in COVID-19 patients. Emerg Microbes Infect 2020;9:761-70. doi:10.1080/22221751.2020.1747363 
29 Gardinassi LG, Souza COS, Sales-Campos H, et al. Immune and Metabolic Signatures of COVID-19 Revealed by Transcriptomics Data Reuse. Front Immuno/ 2020;11:1636. doi:10.3389/fimmu.2020.01636

30 Wu M, Chen Y, Xia H, et al. Transcriptional and proteomic insights into the host response in fatal COVID-19 cases. Proc Natl Acad Sci U S A 2020;117:28336-43. doi:10.1073/pnas.2018030117

31 Gordon DE, Jang GM, Bouhaddou M, et al. A SARS-CoV-2 protein interaction map reveals targets for drug repurposing. Nature 2020;:1-13. doi:10.1038/s41586-020-2286-9

32 Dexamethasone in Hospitalized Patients with Covid-19 - Preliminary Report. N Engl J Med Published Online First: 17 July 2020. doi:10.1056/nejmoa2021436

33 Tomazini BM, Maia IS, Cavalcanti AB, et al. Effect of Dexamethasone on Days Alive and VentilatorFree in Patients with Moderate or Severe Acute Respiratory Distress Syndrome and COVID-19: The CoDEX Randomized Clinical Trial. JAMA - J Am Med Assoc 2020;324:1307-16. doi:10.1001/jama.2020.17021

34 Abu Esba LC, Alqahtani RA, Thomas A, et al. Ibuprofen and NSAID Use in COVID-19 Infected Patients Is Not Associated with Worse Outcomes: A Prospective Cohort Study. Infect Dis Ther 2020;:1. doi:10.1007/s40121-020-00363-w

35 Claeson UP, Malmfors T, Wikman G, et al. Adhatoda vasica: A critical review of ethnopharmacological and toxicological data. J. Ethnopharmacol. 2000;72:1-20. doi:10.1016/S03788741(00)00225-7

36 AMIN AH, MEHTA DR. A Bronchodilator Alkaloid (Vasicinone) from Adhatoda vasica Nees. Nature 1959;184:1317-1317. doi:10.1038/1841317a0

37 McMahon S, Charbonneau M, Grandmont S, et al. Transforming growth factor $\beta 1$ induces hypoxiainducible factor-1 stabilization through selective inhibition of PHD2 expression. $J$ Biol Chem 2006;281:24171-81. doi:10.1074/jbc.M604507200

38 Romero CR, Herzig DS, Etogo A, et al. The role of interferon-y in the pathogenesis of acute intraabdominal sepsis. J Leukoc Bio/ 2010;88:725-35. doi:10.1189/jlb.0509307

39 Leentjens J, Gresnigt MS, van de Veerdonk FL, et al. Adjuvant interferon-gamma immunotherapy in a patient with progressive cerebral Nocardia abscesses. Int J Infect Dis 2017;59:25-8. doi:10.1016/j.ijid.2017.03.013

40 Payen D, Faivre V, Miatello J, et al. Multicentric experience with interferon gamma therapy in sepsis induced immunosuppression. A case series. BMC Infect Dis 2019;19. doi:10.1186/s12879-019-4526-x

41 Wang L, Wang Y, Ye D, et al. Review of the 2019 novel coronavirus (SARS-CoV-2) based on current evidence. Int J Antimicrob Agents 2020;55:105948. doi:10.1016/j.ijantimicag.2020.105948 


\section{Tables}

Due to technical limitations, table 1 is only available as a download in the Supplemental Files section.

\section{Figures}

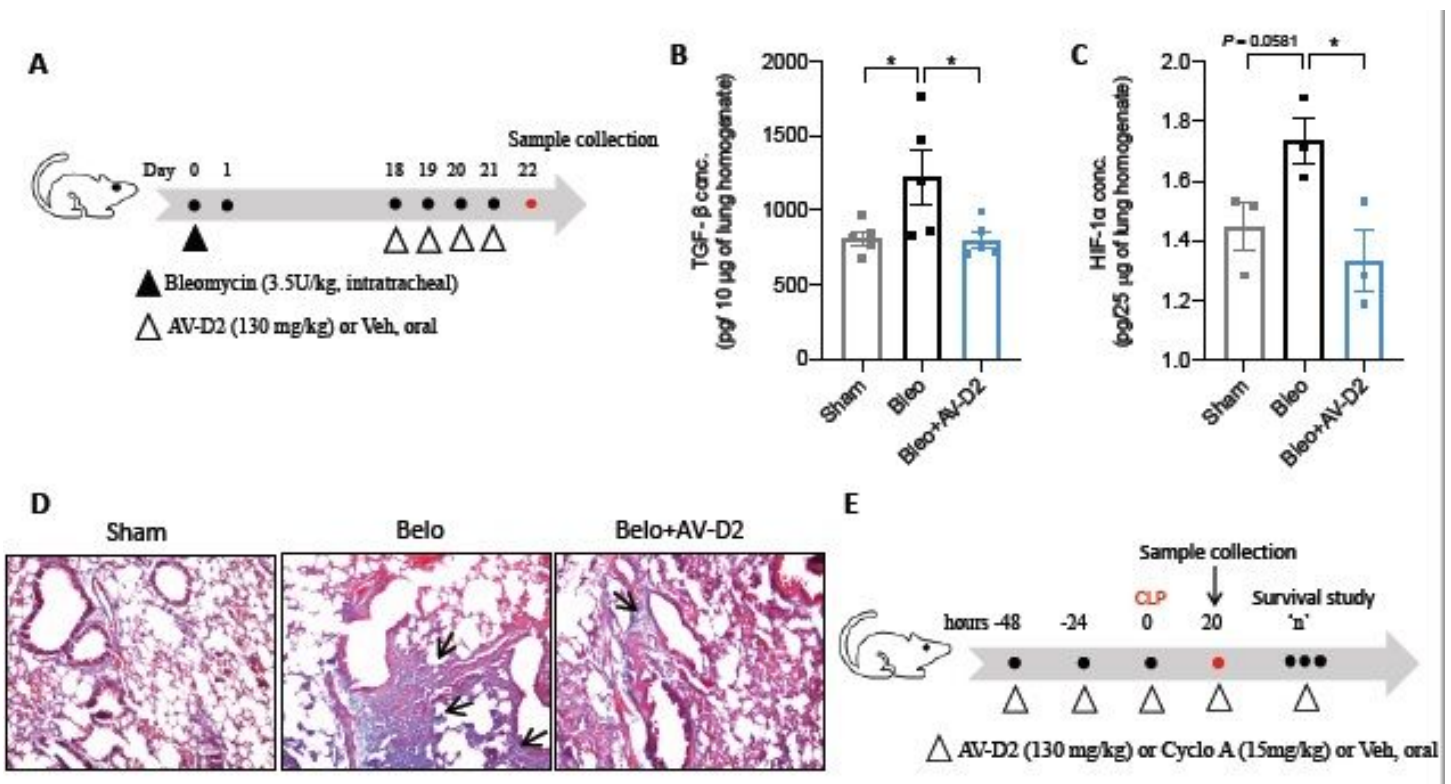

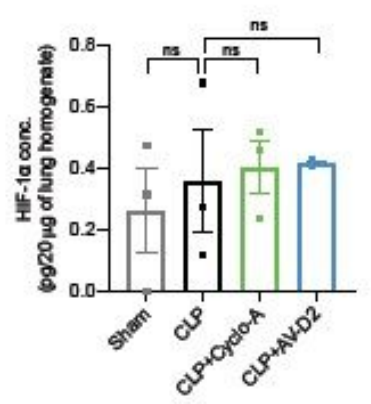

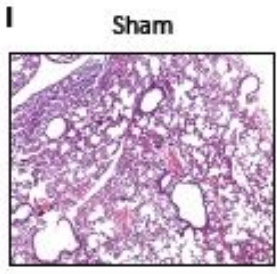

J

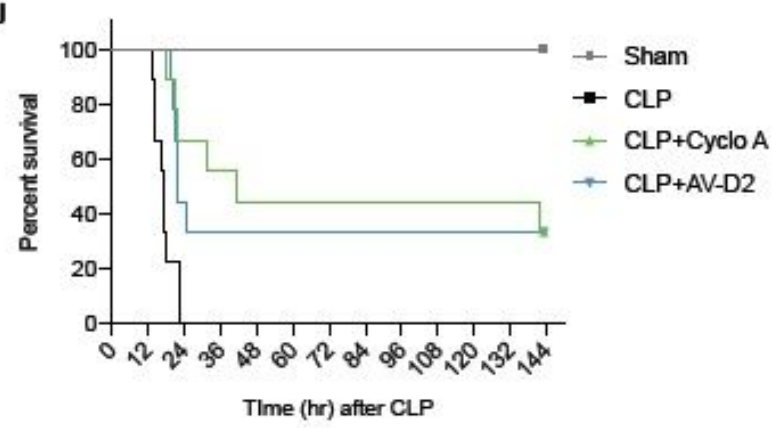

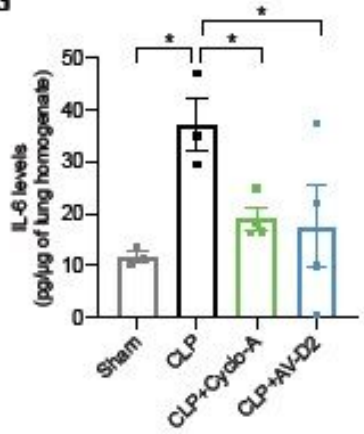
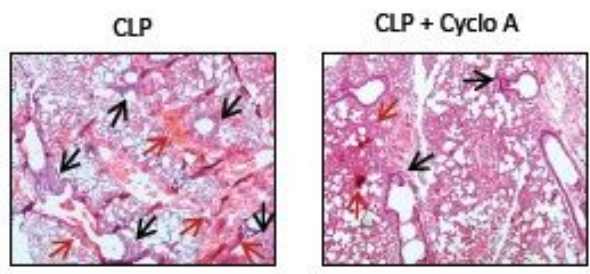

H

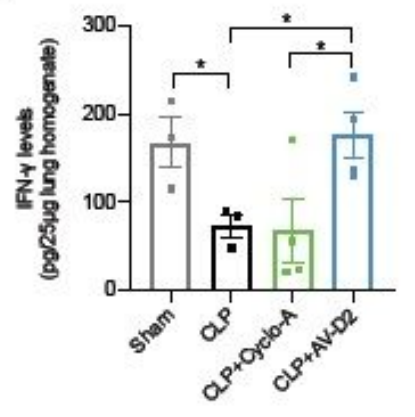

$\mathrm{CLP}+\mathrm{AV}-\mathrm{D} 2$
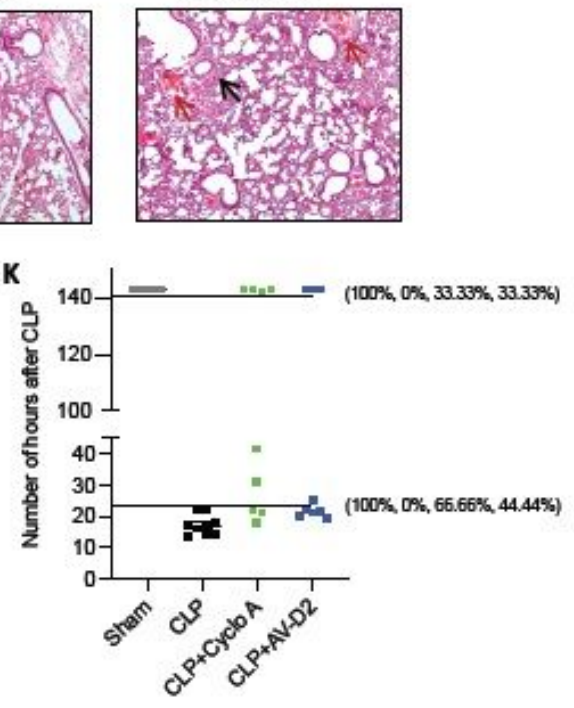

Figure 1 
$A V$ reduces the bleomycin and CLP induced inflammatory features of mice lungs. (A) Schematic representation of bleomycin-induced pulmonary fibrosis model of mice. Male C57BL/ 6 mice were treated with bleomycin on day 0 by the intratracheal route. After genesis of fibrosis features, Bleo mice therapeutically treated with AV-D2 dose $(130 \mathrm{mg} / \mathrm{kg})$ from day 18 to 21 . On day 22, mice sacrificed to collect the sample as described in methods. ELISA for (B) TGF- $\beta 1$ (C) and HIF-1a levels in mice lung homogenate. (D) Representative photomicrographs of fixed mouse lung tissue sections stained with Masson's Trichome (MT) (10X magnification). Positive staining indicated by black arrow. (E) Schematic representation of Cecum ligation and puncture (CLP) mice model protocol. Two days before the CLP surgery, Cyclo A $(15 \mathrm{mg} / \mathrm{kg})$ and AV-D2 $(130 \mathrm{mg} / \mathrm{kg})$ treatment was started, on day 0 CLP was done and after 4 hours of surgery Cyclo $A(15 \mathrm{mg} / \mathrm{kg})$ and AV-D2 $(130 \mathrm{mg} / \mathrm{kg})$ treatment was done and continued till mice survive (' $n$ ' hours). To assess cytokines levels and lung histology after CLP, 3-4 mice from each group was sacrificed after 20 hours of CLP as described in methods. (F) HIF-1a (G) IL-6 (H) and IFN-g levels in mice lung homogenate after 20 hours of surgery. (I) Representative photomicrographs of fixed mouse lung tissue sections stained with $\mathrm{H} \& \mathrm{E}$ ( $4 \mathrm{X}$ magnification) to asses lung inflammation (indicated by black arrow) and associated blood exudation (indicated by red arrow). (J) Survival curve study for 142 hours in CLP induced sepsis mouse. (K) Number of hour's survival plotted for each group showing \% survival after 24 and 142 hours in bracket $(n=5-9)$. Data are shown as mean $\pm S E M$ of three to five mice per group. Significance denoted by ${ }^{*} P \leq 0.05,{ }^{*} P \leq 0.01,{ }^{*} * \mathrm{P} \leq 0.001$ and $* \star \star * P \leq 0.0001$ and determined by ordinary one-way ANOVA) using GraphPad. ns represents the non-significant. Significance of survival curve was determined by log-rank (Mantel-Cox) test using GraphPad. 

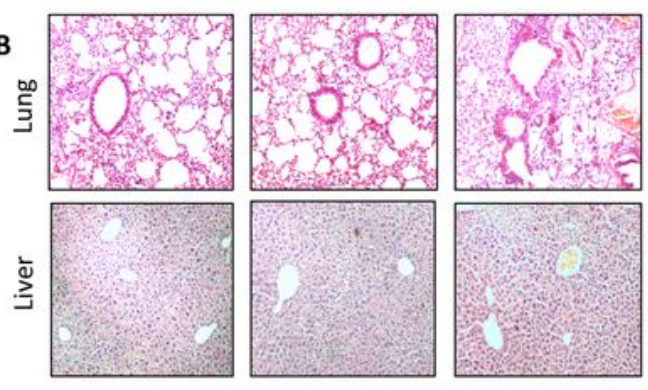

C

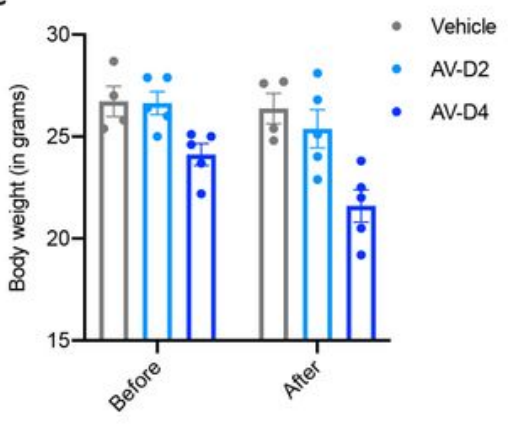

D

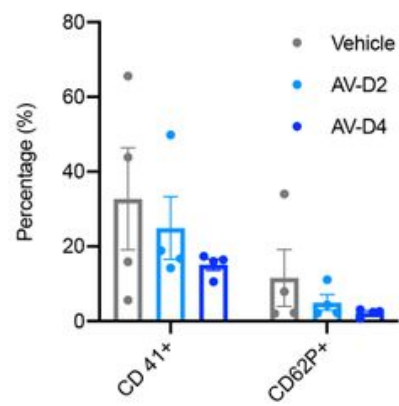

E

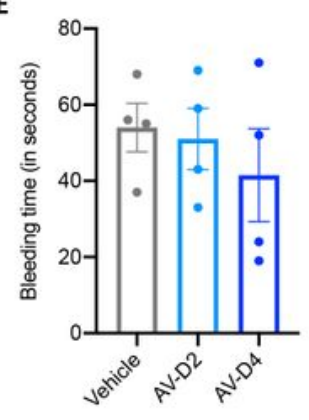

$\mathbf{F}$

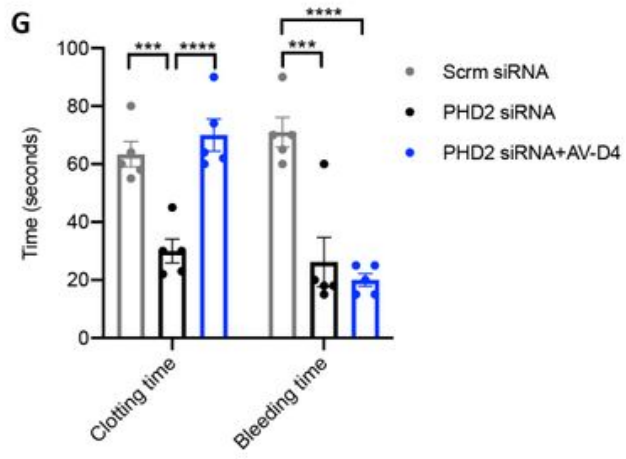

H

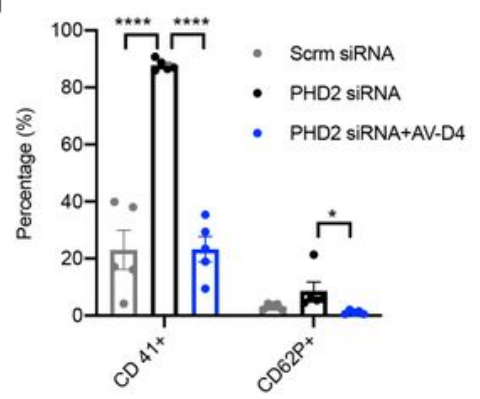

I

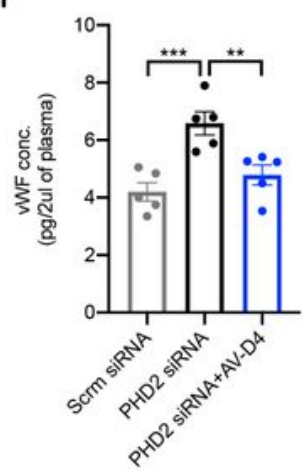

J

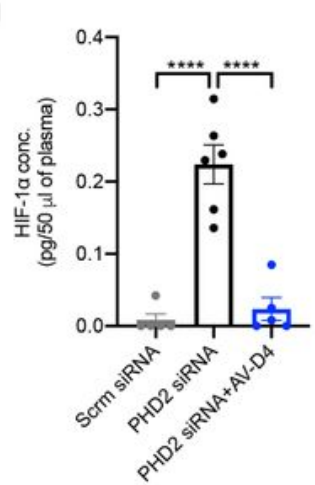

\section{Figure 2}

AV treatment to mice is protective against the blood coagulation phenotype in HIF-1a dependent manner. (A) Schematic representation of $A V$ treatment protocol to naïve $B A L B / c$ mice. $A V$ treatment was given for 4 consecutive days using AV-D2 (130mg/kg) and AV-D4 dose (260mg/kg). (B)Representative photomicrographs of fixed mouse lung and liver tissue sections stained with H\&E (4X magnification) to asses AV mediated any effect on histological architecture in terms of inflammation. (C) Mice body 
weights measured before the start of the AV or Veh treatment and after the completion of treatment (AV or Veh) on day 5th of the protocol. (D) Total (CD41+) and active platelet (CD 62P+) count in mice whole blood (anticoagulated) measured by flow cytometry. (E) Tail bleeding time (in seconds) measured in Vehicle or AV treated mice as per the method described in the protocol. (F) Schematic representation of the siRNA experiment protocol. Scrambled or PHD2 siRNA was given intranasally to mice on days 1, 3, and 5. AV-D4 treatment was started therapeutically from day second and continued till $5 \mathrm{~h}$ day or protocol as described in methods. On day six, mice were subjected to tail bleeding and clotting time assay. (G) Clotting and tail bleeding time (in seconds) measured in mice groups as described in methods. $(\mathrm{H})$ Total (CD41+) and active platelet (CD 62P+) count assessed in mouse whole blood via flow cytometry. ELISA for estimation of (I) VWF and (J) HIF-1a levels in mouse plasma. Data are shown as mean \pm SEM of four to five mice per group. Significance denoted by ${ }^{*} P \leq 0.05$, ${ }^{\star *} P \leq 0.01$, ${ }^{\star \star \star} P \leq 0.001$ and ${ }^{* \star \star *} P \leq 0.0001$ and determined by ordinary one way ANOVA) using GraphPad. 
A

AV up-regulated genes

$P$ Value

1.54613

5.24E 12

8.246:-12

2.436 .10

1366.99

$9.12 E 09$

$5566-13$

1.49E- 10

1376.05

433E-04 Cells and Molecules imolved in local acute inflammatory response WP4493

$4.34 E 08$ Natural biller cell mediated cytotoxicity

1.15E-06 T Tell receptor signaling pathway

9.25E-05 Hematopoietic cell lineage

1.74E-14

$3.306-14$

8.665-14

$7.876-10$

5.13609

641608 Regulation of immune response (G0:005077)

0.0213 mitochondrial outer membrane (60:0005741)

Neutrophil activation inwolved in immune response (60:0002283)

Neutrophil degramulation (60:0043312)

Neutrophil mediated immunity (60:0002446)

$4 E-04$ Fatty acid beta axidation

0.006 T cell activation co-stimulatory signal

CTL mediated immune response against tanget cells

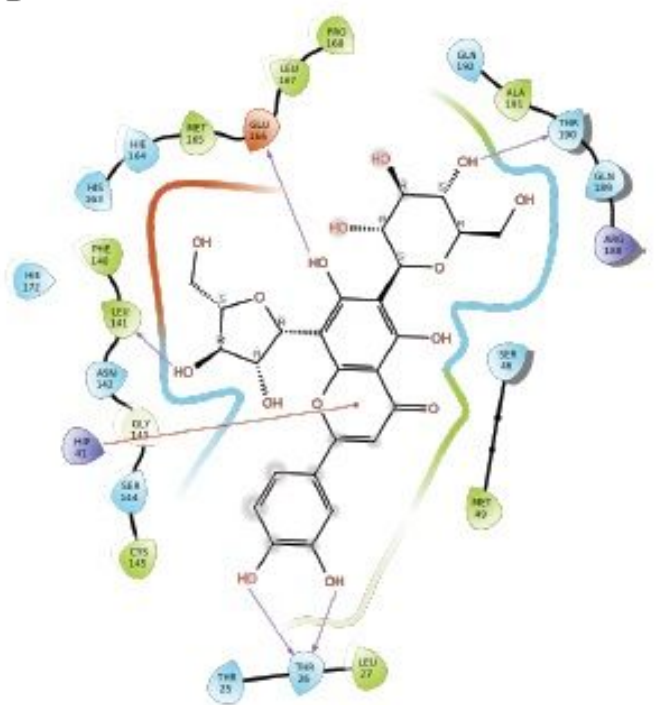

C

\section{AV down-regulated genes}

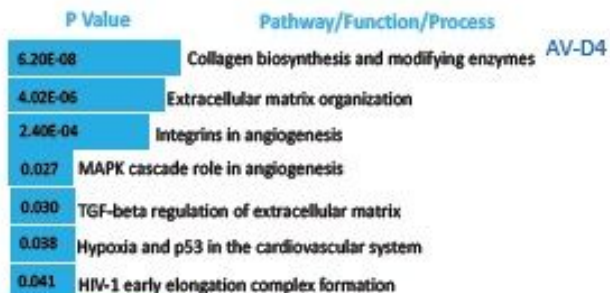

1.43E-05 miRNA targets in ECM and membrane receptors WP2911

0.0031 Robo4 and VEGF Signaling Pathways Crosstalk WP3943

0.0172 ECM-receptor interaction

Q.0430 MAPK signaling pattiway

0.001 TGF-beta regulation of extracellular matrix AV-D2

0.008 MAPK pathwar

a.004 MAPK signalling pathway

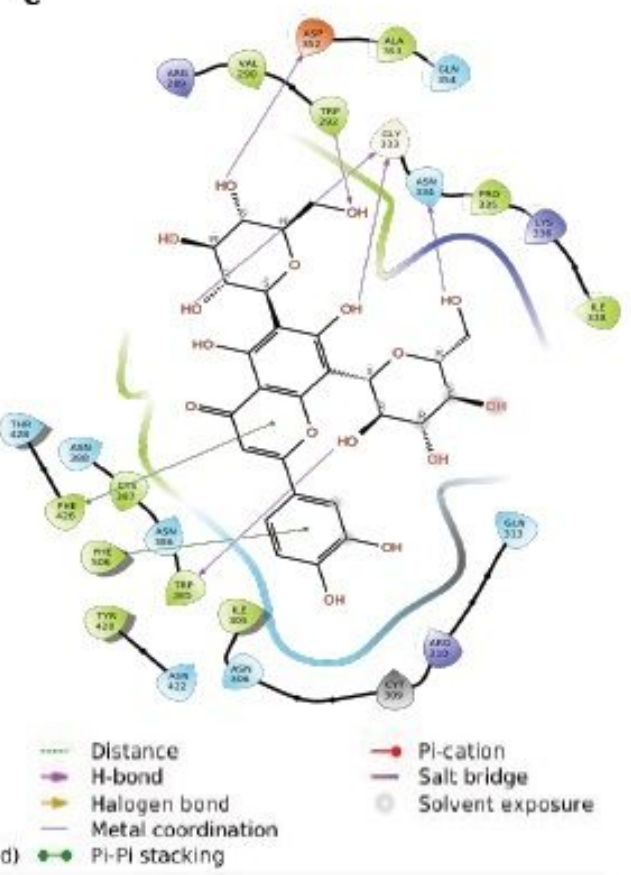

Figure 3

Enriched gene expression pattern of AV treatment may prevent the SARS-CoV2 infection-induced transcriptional changes (A) (A) Enriched pathways and biological process in up-regulated and downregulated genes after $\mathrm{AV}$ treatment compared to vehicle mice group. (B and $\mathrm{C}$ ) Molecular docking interaction of AV's (B) Luteolin-6-C-glucoside-8-C-arabinoside with SARS-CoV2 3CLpro, and (C) Luteolin6,8-di-C-glucoside with SARS-CoV2 NSP14 protein. 


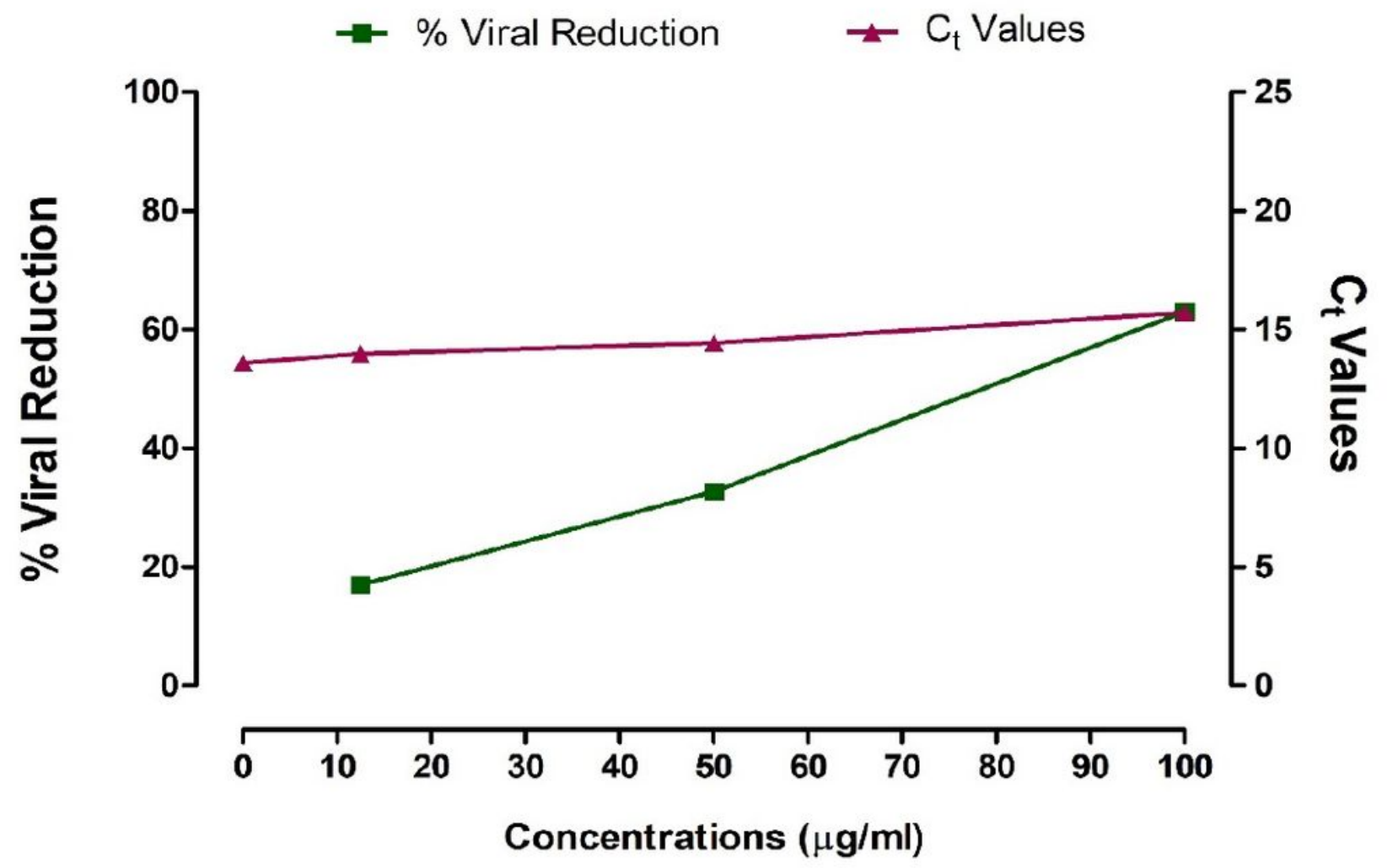

Figure 4

AV treatment inhibits the viral replication in cellular model of SARS-CoV2. Graph showing increase in Viral Reduction (\%) to an increase in Ct Values. Y-Axis (left): Viral Reduction (\%); Y'-Axis (Right): Ct Values; XAxis: Concentrations of AV $(\mu \mathrm{g} / \mathrm{mL})$. 


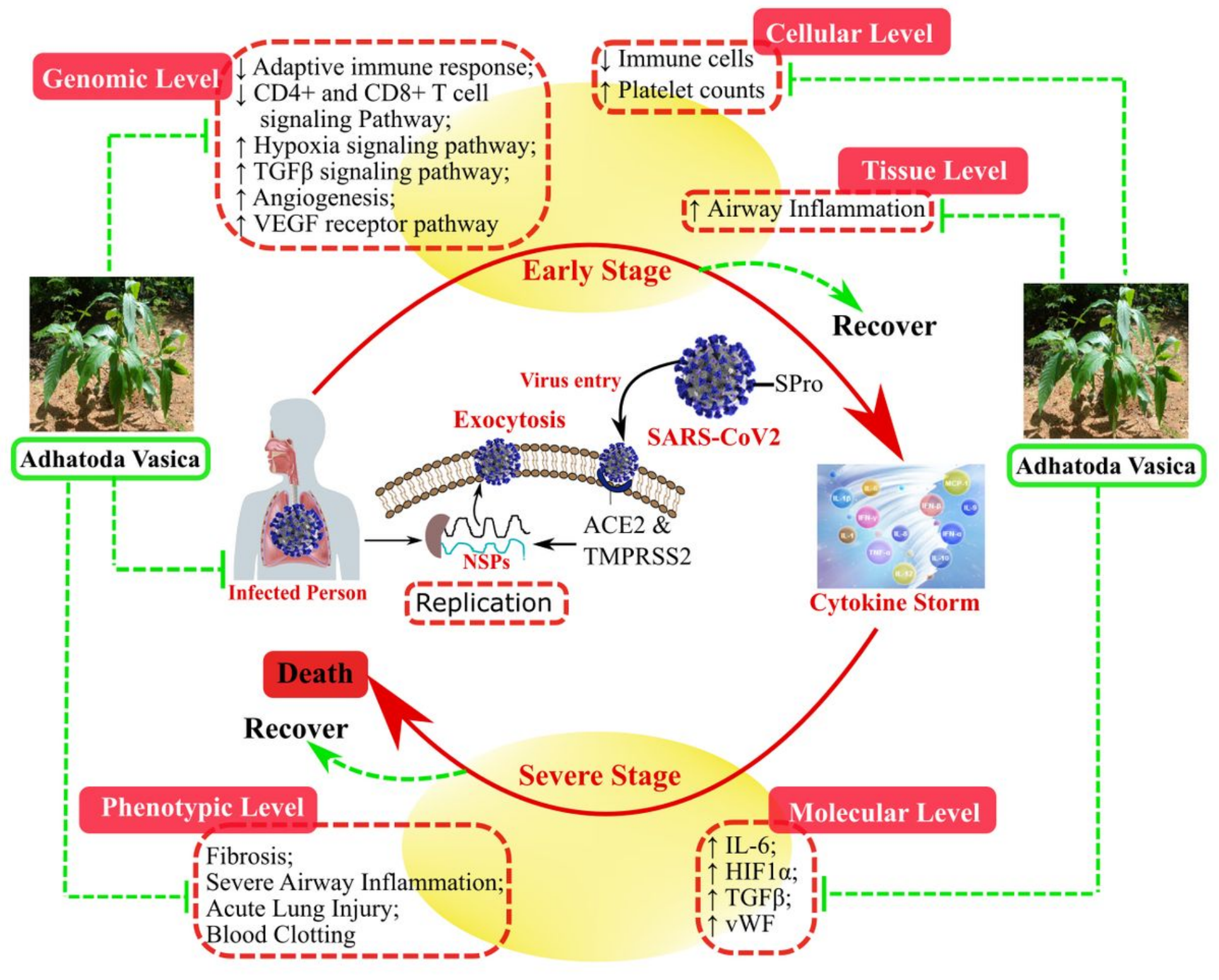

Figure 5

Schematic representation of multidimensional effect of $\mathrm{AV}$ on various markers of immunity hypoxia and inflammatory features of the lung appearing during the early as well as severe phase of COVID-19.

\section{Supplementary Files}

This is a list of supplementary files associated with this preprint. Click to download.

- Supplementarydata.pdf

- Table1.jpg 